\title{
edoc
}

Institutional Repository of the University of Basel

University Library

Schoenbeinstrasse 18-20

CH-4056 Basel, Switzerland

http://edoc.unibas.ch/

\section{Bioreactor based engineering of large-scale human cartilage grafts for joint resurfacing}

\author{
Santoro, Rosaria and Olivares, Andy L. and Brans, Gerben and Wirz, Dieter and Longinotti, Cristina and \\ Lacroix, Damien and Martin, Ivan and Wendt, David.
}

Posted at edoc, University of Basel

Official URL: http://edoc.unibas.ch/dok/A6003825

Originally published as:

Santoro, Rosaria and Olivares, Andy L. and Brans, Gerben and Wirz, Dieter and Longinotti, Cristina and Lacroix, Damien and Martin, Ivan and Wendt, David. (2010) Bioreactor based engineering of large-scale human cartilage grafts for joint resurfacing. Biomaterials, Vol. 31, no. 34. S. 8946-8952. 
Bioreactor based engineering of large-scale cartilage grafts for joint resurfacing

${ }^{1}$ Santoro, R.; ${ }^{2}$ Olivares, A.L.; ${ }^{3}$ Brans, G.; ${ }^{4}$ Wirz, D.; ${ }^{5}$ Longinotti, C.; ${ }^{2}$ Lacroix, D.;

${ }^{* 1}$ Martin, I.; ${ }^{1}$ Wendt, D.

${ }^{1}$ Institute for Surgical Research \& Hospital Management, University Hospital Basel,

Switzerland

${ }^{2}$ Institute for Bioengineering of Catalonia, Spain

${ }^{3}$ Applikon Biotechnology BV, The Netherlands

${ }^{4}$ Laboratory of Biomechanics \& Biocalorimetry, University of Basel, Switzerland

${ }^{5}$ Fidia Advanced Biopolymers srl, Italy

\section{Corresponding author:}

Professor Ivan Martin

Institute for Surgical Research and Hospital Management

University Hospital Basel

Hebelstrasse 20

4031 Basel, Switzerland

Tel: 0041612652384

Fax: 0041612653990

Email: imartin@uhbs.ch

Keywords: Bioreactor, Cartilage tissue engineering, Computational fluid dynamics, Scale-up 


\begin{abstract}
Apart from partial or total joint replacement, no surgical procedure is currently available to treat large and deep cartilage defects associated with advanced diseases such as osteoarthritis. In this work, we developed a perfusion bioreactor system to engineer human cartilage grafts in a size with clinical relevance for unicompartmental resurfacing of human knee joints (50mm diameter x 3mm thick). Computational fluid dynamics models were developed to optimize the flow profile when designing the perfusion chamber. Using the developed system, human chondrocytes could be seeded throughout large 50mm diameter scaffolds with a uniform distribution. Following two weeks culture, tissues grown in the bioreactor were viable and homogeneously cartilaginous, with biomechanical properties approaching those of native cartilage. In contrast, tissues generated by conventional manual production procedures were highly inhomogeneous and contained large necrotic regions. The unprecedented engineering of human cartilage tissues in this large scale opens the practical perspective of grafting functional biological substitutes for the clinical treatment for extensive cartilage defects, possibly in combination with surgical or pharmacological therapies to support durability of the implant. Ongoing efforts are aimed at integrating the up-scaled bioreactor-based processes within a fully automated and closed manufacturing system for safe, standardized, and GMP compliant production of large-scale cartilage grafts.
\end{abstract}




\section{Introduction}

While Carticel ${ }^{\circledR}$ and Hyalograft- $C^{\circledR}$ have been well established in the clinic for the treatment of traumatic focal cartilage defects [1,2,3], no tissue engineered product is currently available to treat large defects or those associated with advanced diseases such as osteoarthritis. Beyond the biological challenges that must be addressed to treat such joint disorders, it remains a significant engineering challenge to generate up-scaled cartilage grafts with dimensions that would be sufficient for the repair of large, advanced, and deep defects.

We previously developed a perfusion bioreactor for seeding and culturing cellscaffold constructs with a clinically relevant thickness $(\approx 4 \mathrm{~mm})$ and demonstrated that highly viable and homogeneous tissues could be generated [4]. However, the diameter of the engineered tissues, representative of constructs typically described in the literature for research purposes (i.e., $\approx 8 \mathrm{~mm}$ diameter), would not be applicable for the treatment of large defects unless multiple plugs were generated and implanted in a surgical procedure resembling mosaicplasty. Therefore, in this work, we scaled-up our perfusion bioreactor system to engineer large-sized human cartilage grafts, in dimensions that would be sufficient for unicompartmental resurfacing of a human knee joint (50mm diameter $\mathrm{x}$ 3mm thick). Computational fluid dynamics (CFD) models were developed to assist in the design of a bioreactor that could generate a uniform velocity profile over the surface of the large diameter scaffold. Experimental validations demonstrated that the developed bioreactor system seeded cells uniformly throughout the large-scale scaffold, and following prolonged culture, supported the generation of viable, homogeneous, and cartilaginous tissue constructs with biomechanical properties approaching those of native 
cartilage. In contrast, constructs generated by conventional manual production procedures were highly inhomogeneous containing a significant non-viable and void region, highlighting the necessity of a perfusion bioreactor-based approach for engineering largescale cartilage grafts.

\section{Methods}

\section{Perfusion bioreactor system}

The bioreactor system was designed to first perfuse a cell suspension directly through the pores of a 3D scaffold, to seed cells uniformly throughout the entire scaffold [5], and subsequently perfuse culture media, to maintain cell viability within the seeded constructs throughout culture [4]. The bioreactor chamber (Applikon Biotechnology BV, The Netherlands) was fabricated from electropolished AISI 316L stainless steel and included four inlet and four outlet ports (Figure 1a) in order to disperse the cell suspension and the culture media uniformly over the large scaffold surface area. Mesh scaffolds were clamped around the outer $1 \mathrm{~mm}$ periphery by a $3 \mathrm{~mm}$ thick Teflon ring (Figure $1 \mathrm{~b}$ ) to prevent culture media from flowing around the scaffold, ensuring flow through the scaffold pores. To provide mechanical support to the delicate mesh and prevent deformation against flow induced drag forces, scaffolds were sandwiched between two stainless steel wire grids (Bopp AG, Switzerland). The chamber was integrated within the bioreactor system shown in Figure 1c, which included a fluid flow pathway for the phase of cell seeding and one pathway for prolonged culture. 


\section{Computational fluid dynamics (CFD) simulations}

Computational models of fluid flow within two proposed bioreactor designs were developed and analyzed using Ansys Fluent 12.1. The first design was based on co-axial single inlet and outlet ports, and second design with four inlet and outlet ports. The scaffold (50mm diameter $\mathrm{x} 3 \mathrm{~mm}$ thick) was simulated as a highly viscous fluid, calculated by setting Darcy's Law and the Poisseuille equation equivalent. The value of the apparent viscosity $\left(\eta_{\mathrm{s}}\right)$ depended on the geometry dimension (scaffold surface area A), properties of fluid (density $\rho$ ) and morphology characteristics (porosity $\phi$, permeability $k$ ).

$$
\eta_{s}=\frac{\pi r^{4} \rho g}{8 A \phi k} \quad \text { Eq. } 1
$$

The properties of the scaffold, a Hyaff-11 non-woven mesh (Fidia Advanced Biopolymers; Italy), were obtained experimentally through mercury porosymetric analysis (average pore size $=68 \mu \mathrm{m}, \phi=91 \%, k=2.13^{*} 10^{-11} \mathrm{~m}^{2}$ ). The continuous phase (culture media), with density approximated as $\rho=1000 \mathrm{~kg} \cdot \mathrm{m}^{-3}$ and viscosity of $\eta=0.001$ Pa·s, was assumed under steady state conditions inside the bioreactor chamber. No slip boundary conditions were applied at the bioreactors walls. Flow rates were $30 \mathrm{ml} / \mathrm{min}$ (corresponding to $18 \mathrm{~mm} / \mathrm{s}$ at the inlet) for cell seeding simulations and $12 \mathrm{ml} / \mathrm{min}$ (corresponding to $7 \mathrm{~mm} / \mathrm{s}$ at the inlet) for culturing simulations. The inlet velocity applied to each of the four inlet ports was divided by four in the multiple-port design as compared to the single inlet port design. Nil pressure was applied at the outlet. An axisymmetric model was developed for the single co-axial port bioreactor whereas a 3D model was developed for the four port bioreactor. 


\section{Cell seeding \& construct culture}

Human Articular Chondrocytes (HAC) were isolated from cartilage biopsies obtained from three individuals (age: 26-60 years) after informed consent and in accordance with the local Ethical Commission. HAC were expanded for 2 passages (8-10 doublings) in basic culture medium (DMEM, 10\% fetal bovine serum, 1mM sodium pyruvate, $100 \mathrm{mM}$ HEPES buffer, 100U/ml penicillin, $100 \mu \mathrm{g} / \mathrm{ml}$ streptomycin, and $0.29 \mathrm{mg} / \mathrm{ml}$ L-glutamine) further supplemented with transforming growth factor- $\beta 1$ (TGF- $\beta 1,1 \mathrm{ng} / \mathrm{mL}$ ) and fibroblast growth factor-2 (FGF-2, 5ng/mL), factors previously shown to increase the proliferation rate and post-expansion redifferentiation capacity of human chondrocytes [6]. A clinically relevant cell seeding density was determined based on the number of chondrocytes that can be obtained from an average cartilage biopsy size obtained for autologous chondrocyte implantation (ACI) procedures $(\approx 280 \mathrm{mg})$ [1], the average chondrocyte yield from a human cartilage digest $(2.5 \mathrm{E}+06$ cells/g tissue) [7], and the number of cell doublings during expansion with two passages ( $\approx 8$ doublings). Based on these calculations, 2.0E+08 HAC were resuspended in basic culture media (90ml) and perfusion seeded into a Hyaff-11 non-woven mesh (50mm diameter $\mathrm{x} 3 \mathrm{~mm}$ thick) at a flow rate of $30 \mathrm{ml} / \mathrm{min}$ for 16 hours within two independent bioreactor systems. Cellseeded meshes were then either harvested and stained with MTT to assess the distribution of cells seeded throughout the scaffold or were further perfusion cultured in the bioreactor (flow rate of $12 \mathrm{ml} / \mathrm{min}$ ) for two weeks to generate a cartilaginous graft. Constructs were cultured in $300 \mathrm{ml}$ chondrogenic media (basic culture media supplemented with $10 \mu \mathrm{g} / \mathrm{mL}$ Insulin, $0.1 \mathrm{mM}$ ascorbic acid 2-phosphate, and 10ng/mL 
Transforming Growth Factor- $\beta 3$ [8]) under hypoxic oxygen levels $\left(5 \% \mathrm{O}_{2}\right)$. In parallel, small diameter constructs ( $\mathrm{n}=2$ per experiment) were also engineered in our previously described "research-scale" bioreactor system [4] (2.0E+06 chondrocytes seeded and cultured on 6mm diameter x 3mm thick Hyaff-11) to serve as controls for the scaled-up bioreactor system. In one experiment, large-scale engineered constructs were also generated by static cell seeding and static culturing methods to compare the bioreactor based approach to conventional manual manufacturing techniques. Engineered constructs were assessed biochemically, histologically, and biomechanically.

\section{$\underline{\text { Histological analyses }}$}

Following the seeding phase, cell-seeded meshes $(\mathrm{n}=2)$ were cut into four sections, rinsed in phosphate buffered saline (PBS), and incubated at $37^{\circ} \mathrm{C}$ for 2 hours with $0.12 \mathrm{mM}$ MTT (3(4,5-dimethylthiazol-2-yl)-2,5-diphenyltetrazolium bromide; Sigma, St. Louis, MO) to assess the distribution of cells throughout the scaffold. Following two weeks of culture, sections of engineered constructs were rinsed in PBS, fixed in $4 \%$ formalin,

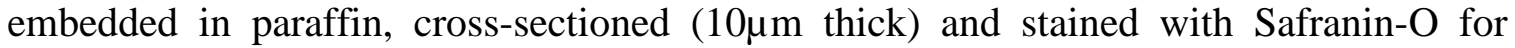
glycosaminoglycans (GAG).

\section{Biochemical quantification}

Engineered constructs were digested with protease $\mathrm{K}$ solution $(1 \mathrm{mg} / \mathrm{ml}$ protease $\mathrm{K}$ in 50mM Tris with 1mM EDTA, $1 \mathrm{mM}$ iodoacetamide, and $10 \mu \mathrm{g} / \mathrm{ml}$ pepstatin-A for 15 hours at $56^{\circ} \mathrm{C}$ ) as previously described [9]. DNA was quantified with the CyQUANT ${ }^{\circledR}$ Cell Proliferation Assay Kit (Molecular Probes, Eugene, OR), with calf thymus DNA as a 
standard. GAG was quantified with the dimethylmethylene blue colorimetric assay, with chondroitin sulfate as a standard [10].

\section{$\underline{\text { Suture pull-out test }}$}

Since the large-scale grafts would likely require fixation with sutures to the surrounding tissue when implanted, pull-out tests were performed to measure the maximal force that could be applied on a suture in the axial direction before pulling out from the construct, as previously described [11]. Sections of the engineered constructs $(\approx 20 \mathrm{~mm}$ length $\mathrm{x}$ $10 \mathrm{~mm}$ wide) were secured at their lower end with a suture (POLYSORB 5-0, coated, braided lactomer 9-1, synthetic absorbable suture with a P-13 needle; Covidien, Mansfield, MA) inserted $1 \mathrm{~mm}$ to $2 \mathrm{~mm}$ above the end of the specimen and fixed with a flying triple knot to a holding hook affixed to the base plate of the mechanical testing instrument (MTS Synergie 100, MTS Systems Corporation, Eden Prairie, MN). The knot from the suture was additionally held by a needle holder to reduce the internal sliding from the knot. The upper end of the specimen was gripped with another suture hung from a rigid metal hook attached to the load cell of the test instrument. The actuator was programmed to apply elongation at a rate of $1 \mathrm{~mm} / \mathrm{min}$ until the suture pulled out of the specimen. The maximal applied force was normalized to the specimen thickness, measured prior to performing the tests, and is reported as $\mathrm{N} / \mathrm{mm}$.

\section{$\underline{\text { Indentation tests }}$}

Biomechanical properties of the engineered constructs were assessed by both dynamic and impact indentation tests [12]. Three specimens were punched out of each construct 
and assessed with five repetitions for each test. Dynamic loading was performed with a MTS Synergie 100 using an indenter tip of radius $1.585 \mathrm{~mm}$. Repeated single sinusoidal cycles at $0.1 \mathrm{~Hz}$ were applied under displacement control to a depth of $\approx 0.1 \mathrm{~mm}$, separated by 50s pauses, shown to be sufficient to allow dimensional recovery. Impact loading was performed with a single impact micro-indentation (SIMI) device, mounted in a rigid load frame. The SIMI indenter pendulum (radius $=500 \mu \mathrm{m}$ ) falls freely, with impact force determined by mass $(1.7 \mathrm{~g})$ and gravity, which provides indentation depths of $\approx 0.1 \mathrm{~mm}$ to $0.2 \mathrm{~mm}$ in healthy cartilage [12]. The motion of the indenter during indentation and rebound is captured by an electromagnetic coil at a sampling rate of $125 \mathrm{kHz}$. The aggregate moduli $\left(E^{*}\right)$ were calculated using equation 2:

$$
E^{*}=\frac{3}{4}\left(1-v^{2}\right)\left[\frac{P_{\alpha \max }}{R^{1 / 2} \alpha_{\max }^{3 / 2}}\right] \quad \text { Eq. } 2
$$

where, $v$ is Poisson's ratio (with $v=0.44$ [13]), $\alpha_{\max }$ is the maximum displacement, $P_{\alpha \max }$ is the force at maximum displacement, and $\mathrm{R}$ is the indenter radius.

\section{Results}

\section{$\underline{\text { CFD simulations }}$}

With the goal of obtaining a uniform cell seeding distribution and homogeneous tissue development, we aimed to design a perfusion bioreactor chamber that could generate a uniform velocity profile over the surface of a 50mm diameter scaffold. Initial concepts for the chamber were based on a simple scaling of a "research-sized" bioreactor, previously designed to house a scaffold $6-8 \mathrm{~mm}$ in diameter [4]. CFD simulations predicted this initial design, which was based on coaxial single inlet and outlet ports, to 
induce significantly higher fluid velocities at the center of the scaffold than towards the periphery, for the range of flow rates applied experimentally in this study (Figures 2\&3). Alternatively, simulations based on the four-port chamber showed the flow to quickly disperse within the main body of the chamber, resulting in a uniform velocity profile over the surface of the $50 \mathrm{~mm}$ diameter mesh (Figures $2 \& 3$ ). The effect of the bioreactor design on the velocity profile at the scaffold surface was quite prominent at the high flow rate applied during the cell seeding phase. As shown by the velocity distributions plotted in Figure 2, for the single-port bioreactor, the fluid reaches a high peak velocity of over $6 \mathrm{~mm} / \mathrm{s}$ near the center of the scaffold and steadily decreases along the radius. In contrast, in the four-port bioreactor, velocity profiles along two different radii (one passing directly under one port and the other passing mid-way between two ports) were similar and quite constant (approximately $1 \mathrm{~mm} / \mathrm{s}$ ), indicating a uniform flow profile over the entire scaffold surface.

\section{Cell seeding distribution}

Consistent with the CFD simulations, which predicted a uniform velocity profile at the surface of the scaffold, MTT staining of the cell-seeded $50 \mathrm{~mm}$ meshes showed a homogeneous distribution of cells throughout the scaffold volume (Figure 4), similar to previous results of perfusion cell seeded small-scale scaffolds [5]. The absence of staining at the periphery of the scaffold verified that clamping of the mesh was effective in ensuring fluid flow (i.e., the cell suspension) through the scaffold pores and preventing flow around the edges of the scaffold. 


\section{Graft handling properties}

Following two weeks of culture, macroscopically, large-scale perfused constructs had a similar appearance as the small-scale perfused constructs, despite the 70-fold scale-up in size (Figure 5a,b). Large-scale constructs were quite stiff such that they could easily be handled with forceps and did not bend under their own weight (Figure 5c). On the other hand, these constructs were flexible enough to readily be bent when applying force with the forceps (Figure 5d), suggesting their potential to be molded to the contours of a condyle during surgical implantation.

\section{Graft composition and structure}

Statically-seeded/statically-cultured constructs had an average of $0.24 \pm 0.07 \%$ GAG per wet weight tissue, but histologically were highly heterogeneous. As shown in Figure 6, statically cultured constructs were encapsulated by a dense layer of cells and were intensely stained for Safranin-O along the outer $0.5-1.0 \mathrm{~mm}$ periphery, but contained a necrotic internal region that was essentially void of viable cells and extracellular matrix. Due to the highly inhomogeneous structure of these constructs, additional analyses were not performed. Constructs seeded and cultured in the large-scale perfusion bioreactors contained similar fractions of GAG as those cultured in the small-scale systems (50mm: $0.15 \pm 0.03 \%$; $6 \mathrm{~mm}: 0.43 \pm 0.05 \%$ GAG per wet weight) and were histologically highly homogeneous (Figure 6b\&c). Cells were uniformly distributed throughout the entire volume of the scaffolds and embedded within extracellular matrix positively stained for Safranin-O. To quantitatively assess the uniformity of extracellular matrix deposition within a large $50 \mathrm{~mm}$ construct, samples $(5 \mathrm{~mm}$ in diameter) were punched from eight 
different locations across the diameter of the scaffold and biochemically assessed for GAG content. Samples across the diameter were found to have similar GAG fractions, with a coefficient of variation of only $27.5 \%$ among the specimens.

\section{$\underline{\text { Graft biomechanical properties }}$}

Following two weeks of culture, the average suture pull-out force for $50 \mathrm{~mm}$ perfused constructs was $0.83 \pm 0.10 \mathrm{~N} / \mathrm{mm}$, which was $20 \%$ of the force previously found for native cartilage $(4.5 \mathrm{~N} / \mathrm{mm})$ [11]. Suture retention test were not performed on $6 \mathrm{~mm}$ constructs due their small size, which physically precluded their testing. The aggregate moduli under impact testing were similar for $50 \mathrm{~mm}$ and $6 \mathrm{~mm}$ constructs (50mm: $1.70 \pm$ $0.19 \mathrm{MPa}$; $6 \mathrm{~mm}: 1.88 \pm 0.49 \mathrm{MPa}$ ), and were approximately $10 \%$ of the modulus of native cartilage [12]. The aggregate modulus of 50mm constructs under dynamic testing was $30 \%$ that of $6 \mathrm{~mm}$ constructs (50mm: $0.32 \pm 0.13 \mathrm{MPa} ; 6 \mathrm{~mm}: 1.12 \pm 0.56 \mathrm{MPa}$ ) and $5 \%$ of the modulus for native cartilage [12]. Cell-free Hyaff-11 meshes were also assessed and found to have a negligible suture pull-out force and negligible aggregate moduli (under both dynamic and impact modes), with values below the sensitivities of the instruments.

\section{Discussion}

In this work, we have developed a perfusion bioreactor system to scale-up engineered human cartilage grafts to a size with clinical relevance for unicompartmental resurfacing of a human condyle. CFD modeling was employed during bioreactor development as part of a rational design strategy, leading to a bioreactor chamber that facilitated uniform cell seeding throughout the large scaffold volume and the generation of a homogeneous 
cartilaginous tissue construct. To our knowledge, this is the first report of engineered cartilage constructs generated in this large-scale.

While one seemingly intuitive design to generate uniform flow over the scaffold would be the inclusion of a flow distributor (e.g., perforated plate) within the chamber, it is likely that a significant fraction of the perfused cells would attach/settle on such a device during the cell seeding phase [5], thereby dramatically reducing cell utilization. As opposed to scaling-up the bioreactor chamber through a trial and error approach, CFD modeling allowed for the efficient assessment and refinement of various chamber geometries during the initial conceptual design stage. Therefore, the bioreactor could be optimized, in term of flow profile, prior to manufacturing costly prototypes and performing time-consuming experimental tests, saving time and resources.

Hyalograft-C, a $2 \mathrm{~cm} \times 2 \mathrm{~cm} \times 0.15 \mathrm{~cm}$ thick Hyaff-11 based cartilage graft, has been shown to provide a clinical improvement to young patients with focal cartilage defects $[2,14,3]$. While the current manufacturing process, based on conventional manual static cell/tissue culture techniques [15], may be sufficient to produce Hyalograft-C in its current size, we speculated that a perfusion bioreactor-based approach could improve the quality of larger sized grafts. Indeed, the data we have presented here are consistent with our previous study [4] showing that the viability, homogeneity, and quality of engineered tissue constructs that are several millimeters in thickness can be dramatically improved when cultured under perfusion vs conventional static culture methods. Moreover, we have also shown that we could generate $50 \mathrm{~mm}$ diameter constructs in the scaled-up bioreactor with similar quality to those generated in our small research-scale bioreactor system, despite the 70 -fold up-scaling $\left(5.9 \mathrm{~cm}^{3}\right.$ vs. $\left.0.085 \mathrm{~cm}^{3}\right)$. 
One of the central questions in cartilage tissue engineering that remains to be answered is "How good is good enough?". In other words, what degree of in vitro maturation is required for a cartilage graft to support or induce a successful repair? Based on ectopic in vivo model systems, Hyaff-11 based constructs that were pre-cultivated in vitro to generate a cartilaginous extracellular matrix had an enhanced capacity to further develop in vivo as compared to those constructs that contained only cells attached to the Hyaff-11 meshes [11,8]. Using a bioreactor-based in vitro model system to simulate aspects of joint loading, Demarteau found that mechanical deformation could increase the synthesis and accumulation of GAG in engineered cartilage constructs, but only if the tissues were sufficiently developed prior to the time of loading [16], suggesting that engineered cartilage tissues may need to possess sufficient mechanical integrity and biological responsiveness prior to implantation.

At the time of its implantation, the Hyalograft-C graft contains chondrocytes attached to the Hyaff-11 mesh fibers, but little to no extracellular matrix, and thus, maturation of the implanted construct (biochemically and mechanically) occurs within the repair site [17], typically taking more than 18 months [18]. While a graft with negligible mechanical properties may be sufficient for filling focal defects, in which the surrounding healthy native cartilage may be sufficient to support joint loading, a graft for resurfacing the lateral or medial condyle would likely need to be at a more advanced stage of maturation to provide a higher degree of functionality. Interestingly, recent midterm follow-up studies have shown poorer clinical outcomes, in terms of defect filling and repair, of larger vs smaller defects treated with Hyalograft-C [14,3]. Based on a short culture period of only two weeks, the $50 \mathrm{~mm}$ constructs engineered in our scaled-up 
bioreactor had $20 \%$ of the suture retention and $5-10 \%$ of the aggregate moduli of native cartilage tissue. If deemed necessary, the culture time could simply be prolonged or culture conditions further optimized (e.g., culture media supplements, flow rate, oxygen tension) to further increase construct maturity. However, a more developed tissue with higher mechanical properties could potentially be challenging to mold to the contours of a knee and may therefore require the engineering of a graft of pre-defined shape. In that case, the design strategy outlined in this work could serve as a foundation to design a bioreactor for seeding and culturing constructs of anatomically shaped grafts.

We have previously reported on the engineering of thin small-scale Hyaff- 11 based cartilage constructs, generated by conventional labor-intensive and manual static cell/tissue culture techniques [19]. In the current study, we have generated cartilaginous constructs with comparable GAG staining and GAG contents, which were 140 -fold larger in size and which were produced by bioreactor-based methods that are amenable to process automation and control. In this context, ongoing efforts are aimed at integrating the up-scaled bioreactor within a fully automated and closed manufacturing system (Figure 7) with the ultimate goal of safe, standardized, cost-effective and GMP compliant production of large-scale cartilage grafts. In conjunction with surgical or pharmacological therapies, which may aid in the durability of the implanted graft, the engineering of human cartilage tissues in this large scale paves the way for grafting functional biological substitutes for the clinical treatment of extensive cartilage defects.

\section{Acknowledgements}


We would like to acknowledge the European Union for financial support (“STEPS”; FP6\#NMP3-CT-2005-500465, www.stepsproject.com).

\section{Figure Legends}

Figure 1. (a) Scaled-up perfusion bioreactor for engineering human cartilage grafts in a size with clinical relevance for unicompartmental resurfacing a human knee joint. (b) Hyaff-11 non-woven mesh scaffolds (50mm diameter x $3 \mathrm{~mm}$ thick) were clamped at the periphery to ensure flow through the scaffold pores. Stainless steel grids provided mechanical support to cell-scaffold constructs during seeding and perfusion culture. (c) The bioreactor system was designed to first perfuse a cell suspension directly through the pores of a 3D scaffold, to seed cells uniformly throughout the entire scaffold [5], and subsequently perfuse culture media, to maintain cell viability within the seeded constructs throughout culture [4].

Figure 2. CFD simulations of fluid flow within the bioreactors under the cell seeding flow rate of 30ml/min. (Top left) In the bioreactor with single coaxial inlet and outlet ports, significantly higher fluid velocities were predicted at the center of the scaffold than towards the periphery. (Top middle) Simulations based on the four-port chamber showed the flow to quickly disperse within the main body of the chamber after exiting each of the four ports, (top right) resulting in a highly uniform velocity profile over the surface of the 50mm diameter scaffold. (Bottom) Velocity distributions at the scaffold surface along the scaffold radii in the two bioreactor designs. The range of fluid velocities in the singleport bioreactor was quite broad, reaching a peak at over $6 \mathrm{~mm} / \mathrm{s}$ near the center of the scaffold and steadily decreasing along the radius. The velocity distribution in the four- 
port bioreactor was assessed along two different radii, one passing directly under one of the four inlet ports $\left(\mathrm{r}_{1}\right)$, and the other mid-way between two inlet ports $\left(\mathrm{r}_{2}\right)$. The velocity distributions were quite similar along $r_{1}$ and $r_{2}$ and were quite constant along the radius. ( $\square$ velocity in single port bioreactor, $\Delta$ velocity in four-port bioreactor along radius $r_{1}, \circ$ velocity in four-port bioreactor along radius $r_{2}$ ).

Figure 3. CFD simulations of fluid flow within the bioreactors under the culturing flow rate of $12 \mathrm{ml} / \mathrm{min}$. Fluid flow within both bioreactors was more uniform at the culturing flow rate than at the cell seeding flow rate, however, the velocities within the single port bioreactor were clearly still significantly higher at the center of the scaffold than at the periphery.

Figure 4. Two sections of the MTT stained meshes following perfusion cell seeding. Cells were homogeneous distributed throughout the large-diameter scaffolds. The unstained periphery of the scaffold reflects the outer $1 \mathrm{~mm}$ region that was clamped in the bioreactor.

Figure 5. Macroscopic views of (a) small-scale perfused cartilage constructs (6mm diameter) compared to (b) large-scale constructs generated in the bioreactor (50mm diameter) after 2 weeks of culture. (c) Large-scale grafts generated in the bioreactor were quite stiff, could be easily handled with forceps, and did not bend under their own weight. (d) However, the grafts were flexible enough to be bent with forceps and could potentially be molded to the contours of a human knee joint.

Figure 6. Safranin-O staining for GAG following 2 weeks of culture. (Left) Large-scale constructs generated by conventional production methods (static seeding and static culture) had a dense layer of cells and GAG along the outer periphery but contained a 
necrotic internal region void of cells and matrix. (Middle) Small-scale constructs (6mm diameter x 3mm thick) generated in our previously described "research-scale" perfusion bioreactor [4] contained cells and Safranin-O stained matrix that were uniformly distributed throughout the scaffold. (Right) Large-scale constructs generated in the upscaled perfusion bioreactor were histologically similar to small-scale perfused constructs, with cells and GAG homogeneously distributed throughout the cross-sections.

Figure 7. Ongoing efforts are aimed to integrate the perfusion bioreactor within a prototype (Applikon Biotechnology BV, The Netherlands) of an automated and closed bioreactor system, with the ultimate goal of safe, standardized, cost-effective and GMP compliant production of large-scale cartilage grafts [20].

\section{Reference List}

[1] Brittberg M. Autologous chondrocyte implantation-technique and long-term followup. Injury 2008;39 Suppl 1:S40-S49.

[2] Kon E, Gobbi A, Filardo G, Delcogliano M, Zaffagnini S, Marcacci M. Arthroscopic second-generation autologous chondrocyte implantation compared with microfracture for chondral lesions of the knee: prospective nonrandomized study at 5 years. Am J Sports Med 2009;37:33-41.

[3] Nehrer S, Dorotka R, Domayer S, Stelzeneder D, Kotz R. Treatment of fullthickness chondral defects with hyalograft $\mathrm{C}$ in the knee: a prospective clinical case series with 2 to 7 years' follow-up. Am J Sports Med 2009;37 Suppl 1:81S-87S.

[4] Wendt D, Stroebel S, Jakob M, John GT, Martin I. Uniform tissues engineered by seeding and culturing cells in 3D scaffolds under perfusion at defined oxygen tensions. Biorheology 2006;43:481-488.

[5] Wendt D, Marsano A, Jakob M, Heberer M, Martin I. Oscillating perfusion of cell suspensions through three-dimensional scaffolds enhances cell seeding efficiency and uniformity. Biotechnol Bioeng 2003;84:205-214.

[6] Barbero A, Grogan S, Schafer D, Heberer M, Mainil-Varlet P, Martin I. Age related changes in human articular chondrocyte yield, proliferation and post-expansion chondrogenic capacity. Osteoarthritis Cartilage 2004;12:476-484. 
[7] Jakob M, Demarteau O, Schafer D, Stumm M, Heberer M, Martin I. Enzymatic digestion of adult human articular cartilage yields a small fraction of the total available cells. Connect Tissue Res 2003;44:173-180.

[8] Moretti M, Wendt D, Dickinson SC, Sims TJ, Hollander AP, Kelly DJ et al. Effects of in vitro preculture on in vivo development of human engineered cartilage in an ectopic model. Tissue Eng 2005;11:1421-1428.

[9] Hollander AP, Heathfield TF, Webber C, Iwata Y, Bourne R, Rorabeck C et al. Increased damage to type II collagen in osteoarthritic articular cartilage detected by a new immunoassay. J Clin Invest 1994;93:1722-1732.

[10] Farndale RW, Buttle DJ, Barrett AJ. Improved quantitation and discrimination of sulphated glycosaminoglycans by use of dimethylmethylene blue. Biochim Biophys Acta 1986;883:173-177.

[11] Farhadi J, Fulco I, Miot S, Wirz D, Haug M, Dickinson SC et al. Precultivation of engineered human nasal cartilage enhances the mechanical properties relevant for use in facial reconstructive surgery. Ann Surg 2006;244:978-985.

[12] Ardura Garcia H, Daniels AU, Wirz D. Dual-mode dynamic functional stiffness of articular cartilage. European Cells and Materials 2008;16:7.

[13] Korhonen RK, Laasanen MS, Toyras J, Rieppo J, Hirvonen J, Helminen HJ et al. Comparison of the equilibrium response of articular cartilage in unconfined compression, confined compression and indentation. J Biomech 2002;35:903-909.

[14] Kon E, Di MA, Filardo G, Tetta C, Busacca M, Iacono F et al. Second-generation autologous chondrocyte transplantation: MRI findings and clinical correlations at a minimum 5-year follow-up. Eur J Radiol 2010;

[15] Pavesio A, Abatangelo G, Borrione A, Brocchetta D, Hollander AP, Kon E et al. Hyaluronan-based scaffolds (Hyalograft $C$ ) in the treatment of knee cartilage defects: preliminary clinical findings. Novartis Found Symp 2003;249:203-217.

[16] Demarteau O, Wendt D, Braccini A, Jakob M, Schafer D, Heberer M et al. Dynamic compression of cartilage constructs engineered from expanded human articular chondrocytes. Biochem Biophys Res Commun 2003;310:580-588.

[17] Hollander AP, Dickinson SC, Sims TJ, Brun P, Cortivo R, Kon E et al. Maturation of tissue engineered cartilage implanted in injured and osteoarthritic human knees. Tissue Eng 2006;12:1787-1798.

[18] Brun P, Dickinson SC, Zavan B, Cortivo R, Hollander AP, Abatangelo G. Characteristics of repair tissue in second-look and third-look biopsies from patients treated with engineered cartilage: relationship to symptomatology and time after implantation. Arthritis Res Ther 2008;10:R132- 
[19] Candrian C, Vonwil D, Barbero A, Bonacina E, Miot S, Farhadi J et al. Engineered cartilage generated by nasal chondrocytes is responsive to physical forces resembling joint loading. Arthritis Rheum 2007;58:197-208.

[20] Martin I, Smith T, Wendt D. Bioreactor-based roadmap for the translation of tissue engineering strategies into clinical products. Trends Biotechnol 2009;27:495-502. 